\title{
COMPUTER STUDIES OF NEUROPHYSIOLOGICAL AND PSYCHOLOGICAL EVENTS
}

\author{
William R. Uttal, * Louella Cook, Hedwig Kasprzak \\ IBM Research Center, Yorktown Heights, N. Y.
}

This paper is neither an exposition of a revolutionary reorganization of neurophysiological research by the application of computers, nor an expedition in bionics (whatever that is). It is rather a presentation of some of the results of some studies in neural sensory encoding and teaching machine research in which we have utilized computers to handle and process our data. The neural studies concern the caudal photoreceptor of the crayfish which was described almost 30 years ago by Prosser (1934), and the evoked somatosensory potential following the route laid out by Dawson (1950). A brief mention of the concept of the teaching machine, particularly as it might be applied to education in the biomedical field is also included. These three problems represent three different types of data processing requirements because of the nature of the data and data rates. They are presented as illustrations of the families of processing problems one encounters in the biomedical field.

\section{Types of Data Processing Problems}

The selection of an appropriate computer for analysis of biological data depends upon the nature of the data. The following list, while not exhaustive, does demarcate a number of important classes of data which are, in general, handled differently by digital computers.

Class 1. Electrophysiological signals in which important component frequencies may be as high as $2-4,000$ cycles per second (cps) and which require analog-to-digital conversion rates over $10,000 \mathrm{cps}$ to assure adequate following of wave forms. This class includes, most obviously, the spike action potentials recorded from neural or muscle tissue. Data rates are quite high and involve the complete attention of a computer for time intervals greater than the actual recording time. In general, the faster binary computers are required to handle this class of data because very short experimental sessions can create very large amounts of data. Often, it is necessary to process the data between successive samples to avoid flooding the high speed core memory of the computer. The data recorded from our crayfish experiments are excellent illustrations of this sort of problem. The spike potentials typically last for a millisecond and a half, and the fast rise times indicate very high frequency components.

*Present address:- University of Michigan, Ann Arbor, Mich. 
Class 2. Electrophysiological and other transduced signals in which the frequency components are very low, varying from a couple of cps to perhaps $50 \mathrm{cps}$. Analog-to-digital conversion rates for Class 2 signals may be very low with samples taken only every 10-20 milliseconds or less. This class includes such signals as the electroencephalogram (including the evoked potentials described here). Simpler converters and lower speed computers are capable of handling this data. In our experiments on the evoked potential, it will be noted that we used the same system as for Class 1 signals. The data were very heavily edited-only one in every ten samples actually being used - and thus approximated the Class 2 situation described here.

Class 3. Real time experimentation with human beings often involve discrete responses which are quasi-alphabetical in nature. The data rates are extremely slow and may require attention only once or twice a second at intervals several seconds apart. Typewriters and other keyboard-like devices may generate this sort of data. Data rates are so low that very large numbers of terminals may be multiplexed and fed into a computer which would respond, for all practical purposes instantaneously to responses from subjects. The teaching machine problem and psychophysical or human learning experiments exemplify this class of data analysis. Nonbinary machines are often particularly suitable for this type of data.

The theme of this symposium is to combine the engineering aspects and the biological aspects of our work. In accordance with this theme, we should like to describe briefly the equipment which has been used to meet the data processing needs of our experiments on man and crayfish. Then, a discussion of the experiments on the caudal photoreceptor of the crayfish will be presented, followed by a brief mention of the work we have done on evoked brain potentials. The details of those experiments were presented at a conference held by The New York Academy of Sciences, in February 1963, and later published in the Annals series (Uttal \& Cook, 1964), and need not be repeated here. A discussion is then presented of the computer tutoring of stenotype.

\section{INSTRUMENTATION}

\section{A. A Data Logging Device for Electrophysiological Recordings}

The terminal device is block diagrammed in FIGURE 1. The unit is composed of four subsystems: a control logic unit; an analog-to-digital converter; a set of coupling amplifiers; and a digital magnetic tape drive which records the data in the format required for entry into the computer. The neural signals are amplified through conventional high gain preamplifiers appropriate to the particular study and then fed into the analog-to-digital converter. In the present system the converter used was an Epsco Transicon Datrac capable of representing the analog input by up to eight parallel 
binary outputs. Upon receipt of a digitization command signal from the control logic, the converter, by a process of successive approximations, coded the analog signal and at the end of the coding period emitted an "end of conversion" pulse. This pulse and the binary code were power amplified through a system of paralleled coupling amplifiers so that the output voltage levels from the transistorized converter were boosted to levels sufficient to drive the vacuum tube circuitry of the digital tape drive. The end of conversion pulse effected the transfer of the binary information to the tape unit, an IBM 727.

The heart of the terminal is the control logic which automatically sequences the series of operations required to make a machine-readable magnetic tape record.

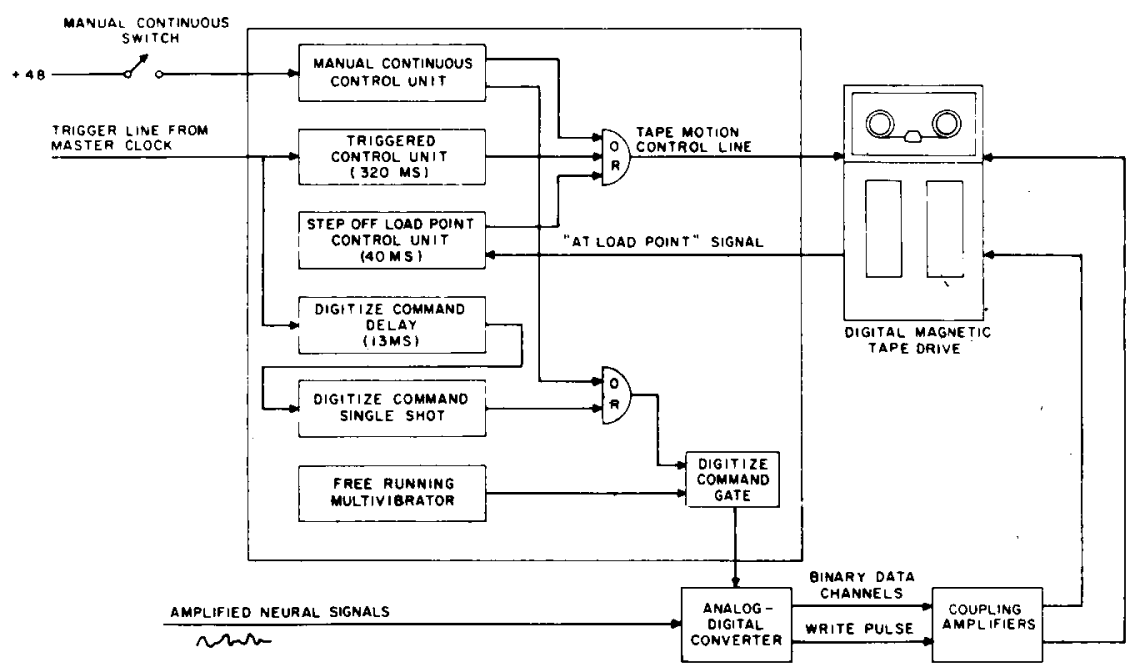

FIGURE 1. A block diagram of the data logging terminal device.

The control logic was composed of a group of electronic circuits which were interconnected in such a way that a small number of input signals produced the necessary voltages to sequence the system through the operations required for the recording of the digital data on the magnetic tape. The circuits utilized in the control logic were conventional computer circuits properly timed for this neurophysiological application.

The control logic may be operated in one of two modes. In the first, a "manual continuous" switch starts the tape in motion and then after the appropriate delay commences the coding conversions at the 12,000 -cps rate. The tape drive and the converter then remain active until the switch is returned to its normal position. In this mode, data may be digitized and recorded from a continuous analog signal for long periods of time. 
In the second mode, the master clock pulse generator emits a pulse which initiates a sequence of control signals which start the tape, command digitizations and stop the tape after a length of time which is predetermined and adjustable by the operator. In this latter mode, a single tape record representing a period of time following a specific event, such as the presentation of a stimulus, can be recorded. Thus, data representing a number of sequential responses may be recorded for later processing on a standard computer.

The control logic is shown in detail in FIGURE 1. Three input lines are required for the complete operation of the control logic. The first simply carries a positive voltage of sufficient level to activate the manual continuous control unit. The second line conducts a brief trigger pulse to the "triggered" control unit activating the system of delays and gates which operate the terminal device in the triggered mode described above. The third input line conducts a signal from the tape unit indicating that the tape is at load point (i.e., the position taken by the tape when the operator first places the tape reel on the 727), automatically initiating a pulse from the "step off load point" control unit which advances the tape a few inches. This operation is required in order that the computer will not miss the first few samples of recorded data.

Three output lines from the control logic conduct signals required for the operation of other components of the system. The tape motion control line, when activated, determines the time period in which the tape will be in motion. The "digitization command" line conducts a series of pulses each of which causes the converter to carry out one conversion, and the feedback line which can be connected to peripheral equipment and can be used for triggering stimuli after the delay sufficient to bring the magnetic tape to full speed.

The single shots and the free running multivibrator which control the durations and delays of the various control signals are all adjustable over wide ranges. As an example, there was usually a $13 \mathrm{msec}$. delay to achieve full tape speed before the digitization commands began and the $320 \mathrm{msec}$. duration used in the evoked potential work was typical of the triggered epoch used in the second mode of operation.

The two logical "or" circuits shown were necessary in order to allow any of several different control signals to cause tape motion and to allow either the manual continuous or the triggered single shot to gate the output of the $12,000 \mathrm{cps}$ free running multivibrator.

To illustrate the operation of the control logic, we may consider the circuitry involved in the triggered mode. The master clock pulse, generated by the stimulation circuitry (the stimulus generator is described elsewhere (Uttal, 1960), triggers two separate inputs. The first is the triggered control unit which produces a $320-\mathrm{msec}$. output pulse which is fed through the tape motion control "or" circuit causing the magnetic tape to move. The second is a delay device which delays the master trigger for $13 \mathrm{msec}$. before activating 
the digitize command single shot. The digitize command single shot then emits a pulse which lasts for $307 \mathrm{msec}$. and during that period, enables the digitize command gate to pass the signals generated by the free running multivibrator. The master trigger delay is required so that the tape will be moving at full speed before any data are written. During the 307 -msec. period, data are written at the 12,000 -cps rate at which the free running multivibrator operates. Simultaneously, at the end of this period the tape motion stops and the digitize command gate is closed to further signals from the free running multivibrator. The system is now ready to be activated by the next master clock trigger after an interval chosen by the experimenter. The manual continuous mode of operation is similar except that the period of operation depends upon the length of time the manual continuous switch is kept closed.

After sufficient data have been recorded, the magnetic tape is removed from the 727 and sent off for processing on a suitably programmed digital computer.

The power and versatility of such a system may be exemplified by the fact that in every session with a subject in the evoked potential study, $17,280,000$ bits of information were generated which had to be handled in one way or another by the computer; yet during the same day, the terminal device was also being used to record equally large amounts of data from the crayfish experiments.

\section{B. The Low Speei) Quasi-AlphabeTical Multipi.exer for Psichologilal. Resfarchand Comprter Titoring;}

The following paragraphs describe some of the design objectives of our buffered multiplexer:

1. Full buffering. For teaching machine and other real time applications a time delay of any significant amount between the input of information from the human operator and the response from the computer can be detrimental to system performance. Therefore, the usual system of inquiry stations used on the 1410 system (as an example) is inadequate for this type of problem because only two buffers are used. Many typewriters are assigned to each buffer and queuing situations could develop which might disastrously lengthen the waiting time for each operator. Therefore, a fully buffered system was specified in which each typewriter was connected to a small memory unit which could perform the buffering operation. (As used in the present context, the term buffer refers to the storage element synchronizing the operation of a high speed system on one side with a low speed system on the other in a duplex or two directional fashion.)

2. Modularity. The buffer multiplexer system described in this report was designed as an experimental tool and, therefore, considerable flexibility was desired in the number and types of terminals which could be connected. It was also thought that the variability of future systems applications suggested 
a modular type of device in which the number of terminals added to the system was completely variable. Thus, the buffer units for each terminal connected to the multiplexer were designed as self-contained units which could be added one by one to the system. A rather artificial maximum of a single gate of SMS (Standard Modular System) circuitry was set in order that the installation of each additional buffer package would require only minimum interchassis connections.

3. Complete format and character control. The typewriter terminal in particular was designed for full computer controlled format. No control tape or ribbon is used and all control and type characters are present. Not only is there a full upper case and lower case alphabetic repertoire, but the computer is also able to space, backspace, tabulate, and return the carriage. Multiple lines of printout are possible using the end of line and end of message features which will be described later.

4. Special terminals. The multiplexer was designed so that many special features could be easily added and used. Thus, the buffer units themselves were more closely associated with the individual terminal unit than with the multiplexer in order that different types of buffers could be used if required. Some of the special terminals added are: $(a)$ a real time clock; $(b)$, a special terminal to teach stenotypy; and $(c)$ a random access audio system.

5. Interchangeability. The design of this system was also motivated by a criterion of interchangeability between many different computer systems. The device is connected to the 1410 through a tape adapter unit (IBM 1414-II) which is now standard on many IBM computers. The entire buffer multiplexer can be disconnected by means of a single cable and then reconnected to any other computer using this standard interface. Within this same context it should be noted that other than a few minor timing changes in the 1414-II to avoid useless delays, no changes of any kind were made in the 1410 system to which this device was attached.

FIGURE 2 is a block diagram of the buffered multiplexer logical organization. The device itself is connected to an IBM 1410 data processing system through an IBM 1414-II tape adapter unit in which a few minor timing changes had been made. These were required because the 1414-II usually drives a group of 107330 magnetic tape units and start and stop times of the tape mechanisms are considerable and had to be accounted for before data flow could commence.

The 1414-II now feeds information and control signals into the multiplexer, a single gate of SMS circuitry, which operates as a signal distribution and control unit. The select line system of the tape adapter unit is used to switch in one of the 10 different input-output terminals.

To expand the multiplexer to more than 10 different terminals, a slightly different select system would be required. This could be formed by a onecharacter (six-bit) storage unit which would then satisfactorily address as many as 63 multiplexed terminals. The multiplexer contained, in addition to 


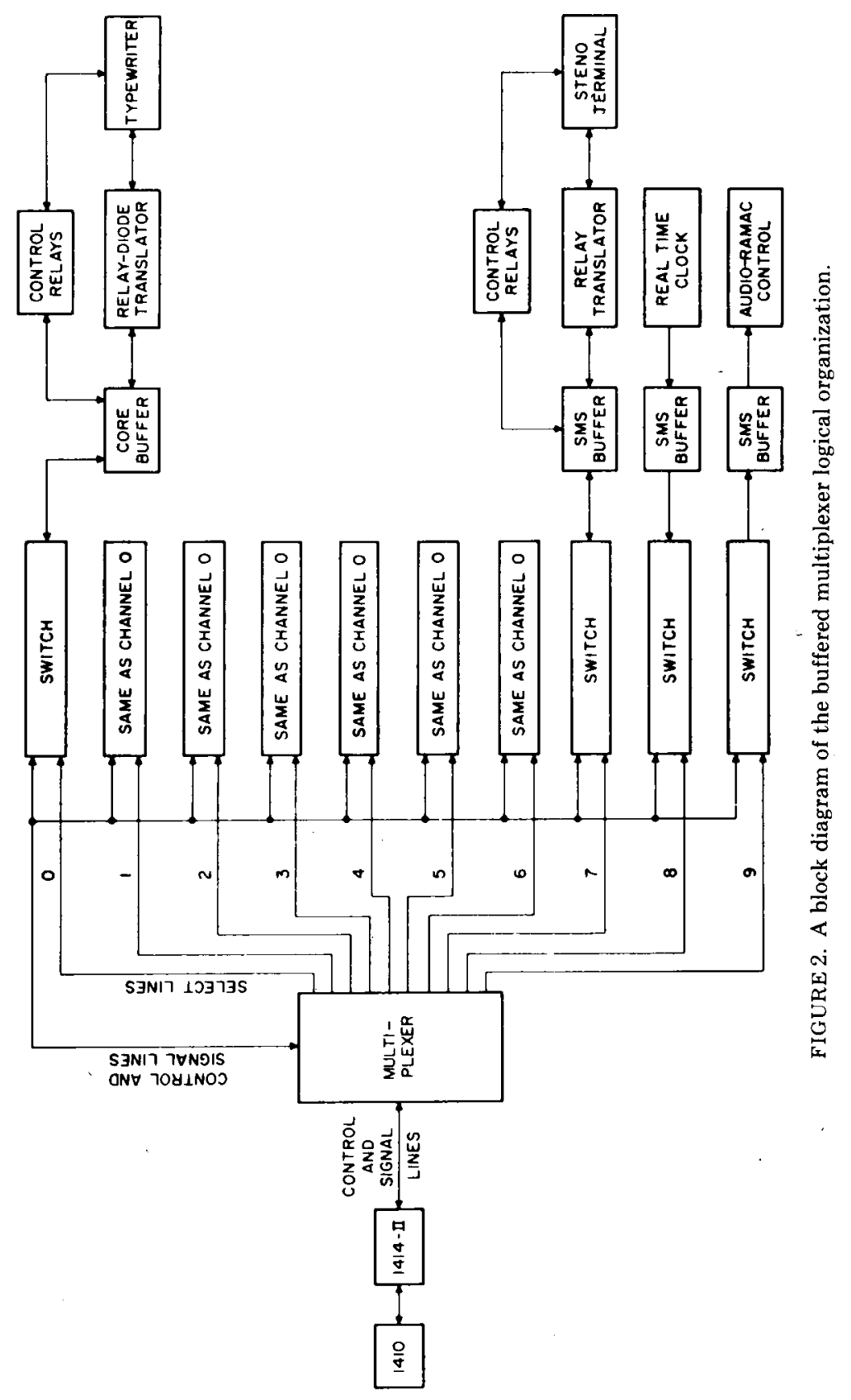


the switching circuitry, coupling circuitry to match voltage levels between the computer and the buffered multiplexer. Some special units which were used commonly in all terminals, such as the $240 \mathrm{kc}$. and $20 \mathrm{kc}$. oscillators, parity generating circuitry for information flowing back into the computer and other necessary circuits which simulated certain control signals usually coming from the tape unit, were also on the multiplexer gate.

Each actual element of the electronic switch was wired on the gate associated with each terminal. Select line signals from the multiplexer activated a number of "and" gates which thus allowed control and signal lines to pass to and from that and only that terminal buffer.

Appropriate control circuitry was necessary in each terminai to sequence the operations and indicate to the computer and state of the system in order that read and write operations could be initiated at the appropriate time. "Not ready" conditions could be caused by a number of states which might differ from one type of terminal to the next. For example, the clock could be in a state of transition from one time count to the next or a student might not yet have released an answer to the computer at the typewriter. Sufficient time delays and appropriate inhibiting circuits were necessary to account for the very considerable amount of time required to actuate a relay compared to the electronic speeds of the computer.

\section{Experimental Results}

\section{A. An Example of Class 1 Data Processing- The Caudal Photoreceptor of the Crayfish}

The problem addressed in this study is the determination of the coding function relating the intensity of monochromatic stimuli to the magnitude of an electrophysiological response in a simple invertebrate photoreceptor. For a number of reasons the crayfish has proven to be an extraordinarily useful animal for this type of research. In addition to the anterior compound eyes which have been shown to have a spectral sensitivity curve of the iodopsin type (Kennedy \& Bruno, 1961), it also has a simple two-cell photoreceptor (Prosser, 1934) in the sixth caudal ganglion which appears to have a spectral response curve of the rhodopsin type (Uttal \& Kasprzak, 1962; Bruno \& Kennedy, 1962).

\section{Procedures}

Stimulation. The caudal photoreceptor was stimulated by narrow bands of light produced by a Bausch and Lomb $250 \mathrm{~mm}$. grating monochromator and focused on the sixth ventral ganglion - the structure containing the as yet unidentified receptor tissue. Exit and entry slit widths were kept constant at $1 \mathrm{~mm}$. to control the purity of the stimulating light. This width meant that our monochromatic light actually was a narrow band of wave length 
$6.6 \mathrm{~m} \mu$ in width. An equal energy spectrum of approximately $3 \mu \mathrm{w} . / \mathrm{sq} . \mathrm{cm}$. was generated by regulating the current applied to the ribbon filament lamp and calibrated daily by measuring the output of a calibrated Reeder thermopile with a microvoltmeter.

Because of the extremely long time constants of the response, the light pathway was arranged to be interrupted manually. A cell containing distilled water was also placed in the light pathway to filter out infrared radiations which might otherwise have caused local heating.

Recording. Simple and conventional response detection techniques were used throughout the experiment. The active electrode (of 0.005 -inch platinum wire) was formed into a hook and was used at the connective between the fourth and fifth abdominal ganglia to simply lift the entire nerve cord above the remaining tissue. An alligator clip was clamped to abdominal muscle tissue to serve as an indifferent reference electrode. The signals were conveyed to a Tektronix 122 preamplifier and thence to an oscilloscope. The vertical output of the oscilloscope was fed into another power amplifier which elevated the signal voltage to a peak-to-peak output of 10 volts and clipped the noise below an adjustable threshold level. The output of this amplifier was fed into the data logging unit. The entire system is shown in block diagram form in FIGURE 3.

Data recording was controlled by a clockwork timer which allowed the amplified neural signal to be recorded for a predetermined length of time. This data logging unit has been described above.

Data analysis. The data recorded on the magnetic tape were then processed on an IBM 7090 computer under control of several special computer programs specifically designed for the analysis of our recordings.

The typical program performed the following three-stage operation upon the raw data:

1. The raw data were read into the computer and inspected by a peak detection program. These raw data were essentially in the form of a table showing the value of the analog voltage 12,000 times a second. The peak detection program first reduced this enormous table to a smaller one which tabulated the significant measurements of each individual spike potential. Thus, a new table was formed in which each sequential spike was numbered, its amplitude tabulated, and the time at which it occurred also indicated.

2. Two tables indicating the distribution of amplitudes and the distribution of intervals between sequential spikes were then tabulated.

3. Two histograms were automatically plotted by the computer presenting the tabular information in stage 2 in a pictorial form which was ideally suited for visual or simple statistical evaluation.

Because pooled interval data from many fibers rapidly approach a random distribution as the number of fibers increases (as described by Cox \& Smith, 


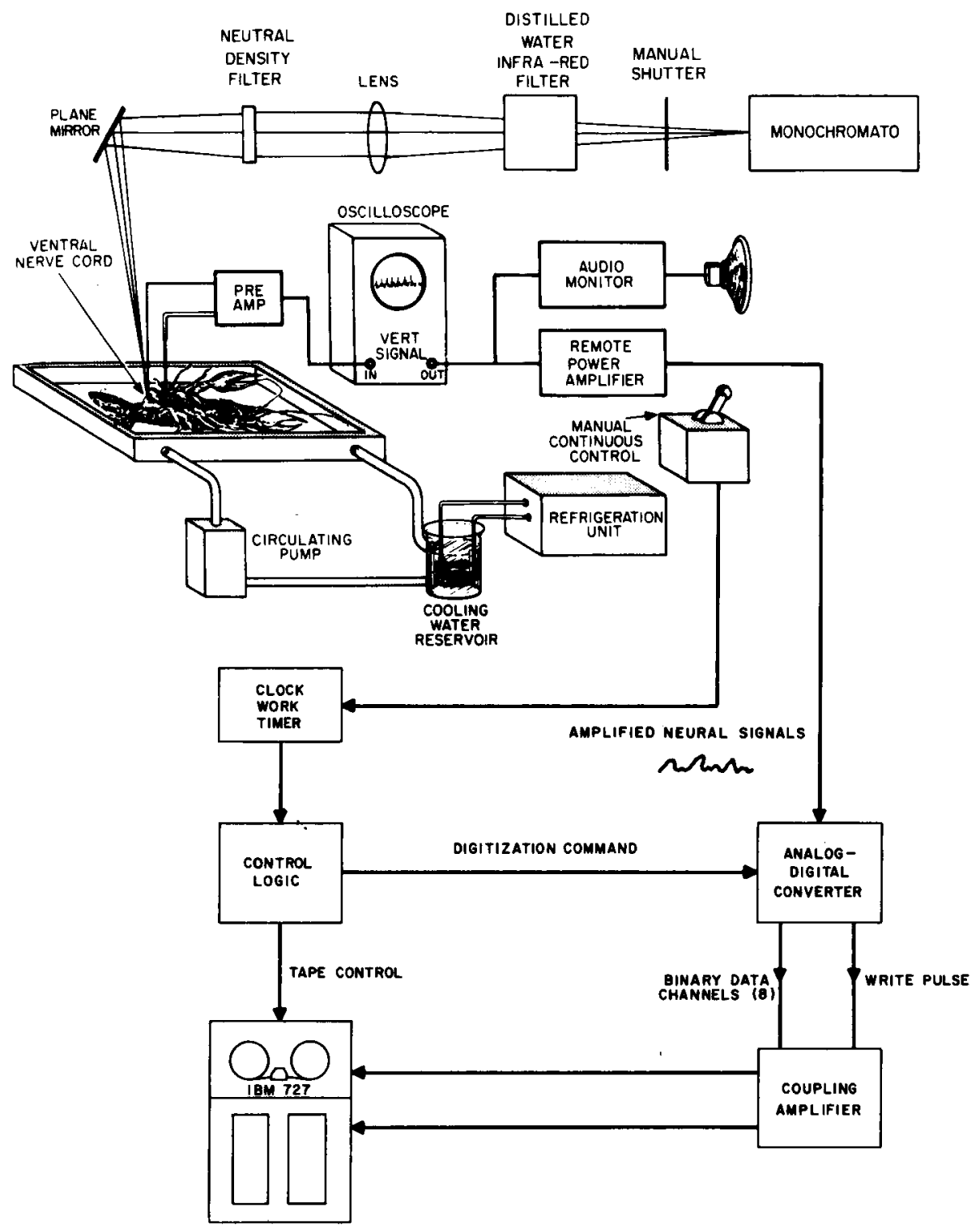

FIGURE 3. A block diagram of the crayfish experimental configuration. 
1954), the amplitude data are more applicable to present experiments and have been used exclusively for the determination of our results. This pulse amplitude discrimination technique is similar to that suggested by Hichar (1959) and Stark (1959). The usual measure of response was a simple summation of the activity within the bandwidth associated with light sensitive fibers. The width of the band associated with the highest intensity of stimulation was used to determine the range of included values. It should be pointed out that this pulse height discrimination technique is but one of a family of analytical techniques which have been programmed for use with this data logging unit. As mentioned above, statistics of intervals were also available in any given experimental session. Many thousands of spike action potentials were examined in both time and amplitude dimensions and a complete tabulation of all responses was available. In this way signals could be detected within considerable amounts of additional noise.

Experimental design. The relationship between stimulus intensity and response magnitude was determined for white light and 10 equally spaced center wave lengths of narrow bands of light. The center wave lengths of the bands chosen were $420,440,460,480,500,520,540,560,580$, and 600 millimicra.

As described in an earlier paper (Uttal \& Kasprzak, 1962), the turn on and turn off latencies and the general time course of the response of the

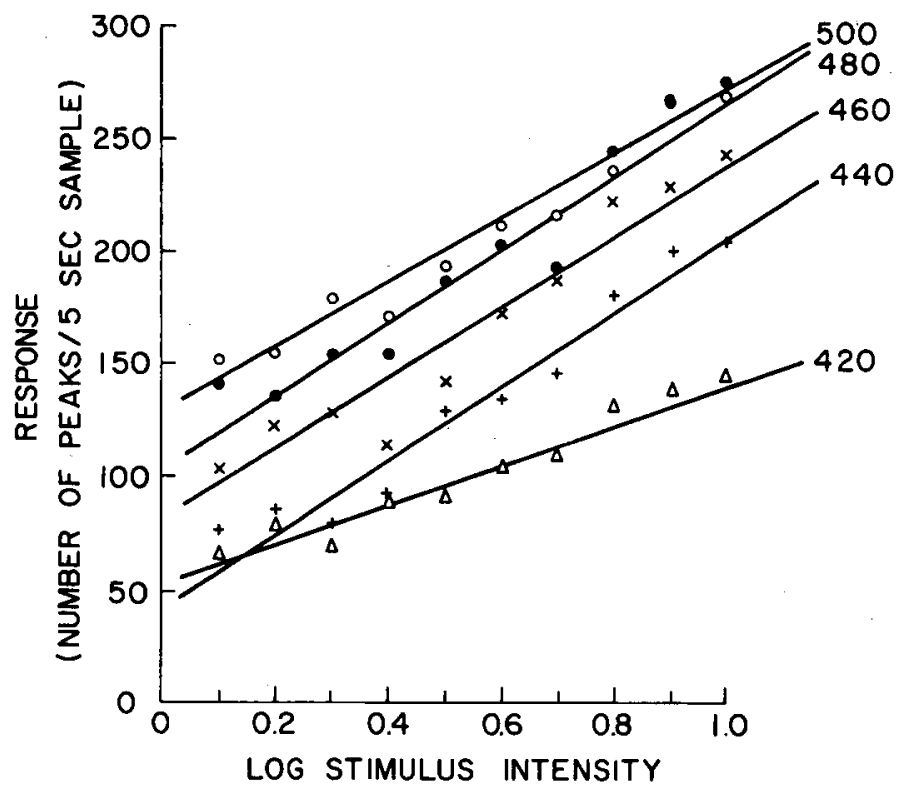

FIGURE 4. The results of the experiment in which the intensities of monochromatic stimuli were varied and response amplitude measured. Results for $420,440,460,480$, and $500 \mathrm{~ms}$. 


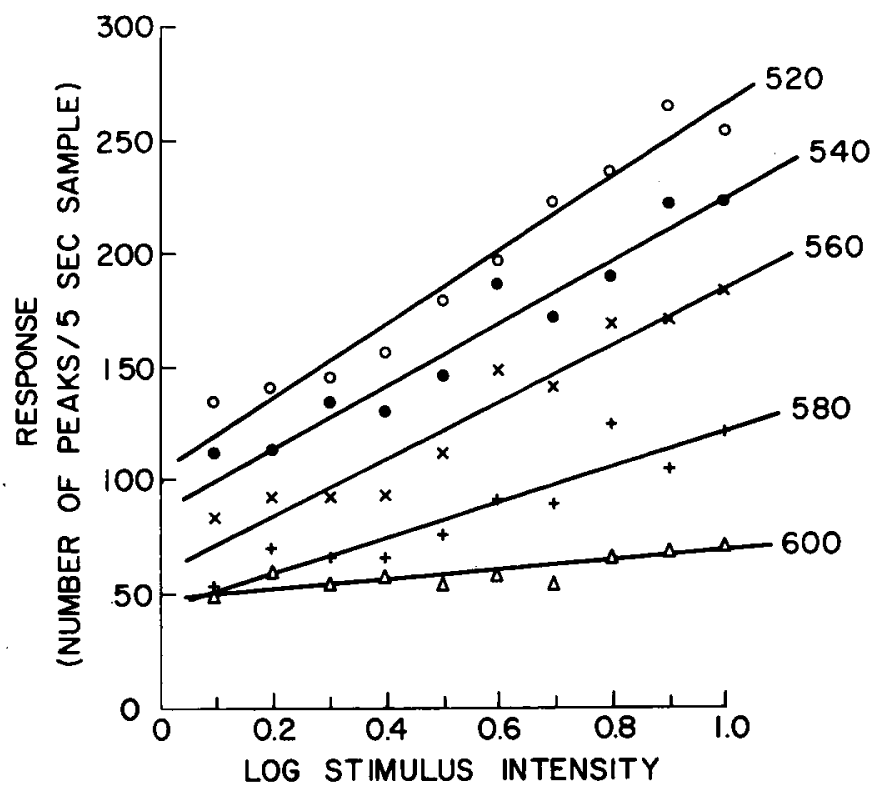

FIGURE 5. The results of the experiment in which the intensities of monochromatic stimuli were varied and response amplitude measured. Results for 520, 540, 560, 580, and $600 \mathrm{~ms}$. (The results have been separated to avoid overlap of nearly adjacent points:)

crayfish photoreceptor are extremely long. The amount of adaptation of the response to the stimulus was very small and remained stable over a very long period of time (one to two minutes). For these two reasons it was possible to choose the recording interval in the following fashion: after a 15-minute dark adaptation period, stimulus wave length and intensity were selected by adjusting the monochromator and selecting a filter density. The stimulus was turned on by opening the shutter. In approximately 15 seconds when the response was judged to be at its peak value, a sample of the signal was recorded on the magnetic tape. The length of the sample was determined by a clockwork timer; it was five seconds for monochromatic light and two seconds for white light. Stimulus intensities were then presented in a constrained random order - random selection without replacement until all intensities had been measured. A new wave length was then selected in a similar constrained random order and the procedure repeated. The crayfish was then discarded and a new animal used for the next two selected wave lengths. Fifteen measurements were made on separate crayfish of the activity at each intensity level for each color and averaged to give each point, shown in FIGURES 4 and 5. 


\section{Results}

FIGURES 4 and 5 show the results of the experiment for the selected monochromatic stimuli. (The data have been drawn on two graphs to avoid confusion between nearly overlapping curves.) The individual stimulus response curve for each wave length is seen to be fit relatively well by a straight line. Such a fit implies that, for our experimental conditions, a logarithmic function best describes the relationship between the two variables.

FIGURE 6 shows the analogous results for the situation in which white light was used as the stimulus and we see that these data are also well fit by a straight line on semilogarithmic coordinates. These results differ somewhat from a measurement for white light which was reported in the earlier paper (Uttal \& Kasprzak, 1962). This difference is due apparently to local heating which had been caused by infrared radiation included in the light and has been obviated by the use of the distilled water cell in the light pathway. The curve now appears to approach a logarithmic function rather than the power function previously found.

A constant response criterion of 145 units of activity was then used to determine the spectral sensitivity function. The relative amount of ambient energy was computed for each wave length by determining the stimulus intensity at the intersection of the straight line fitted to the data points with the 145-unit axis. These values were plotted superimposed upon Wald and Brown's (1958) data which show the spectral sensitivity curves for psychophysical, theoretical, and biochemical measures of the rhodopsin of the scotopic component of human vision. This plot is shown in FIGURE 7 and generally confirms both our earlier measurement with a constant energy

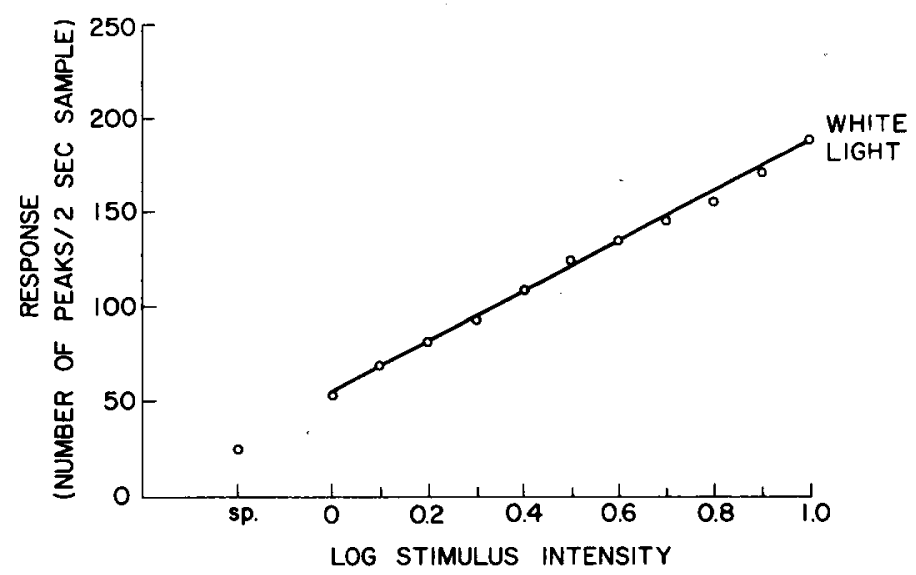

FIGURE 6. The stimulus intensity response amplitude relation for white light. Note that these results are for two-second samples. 


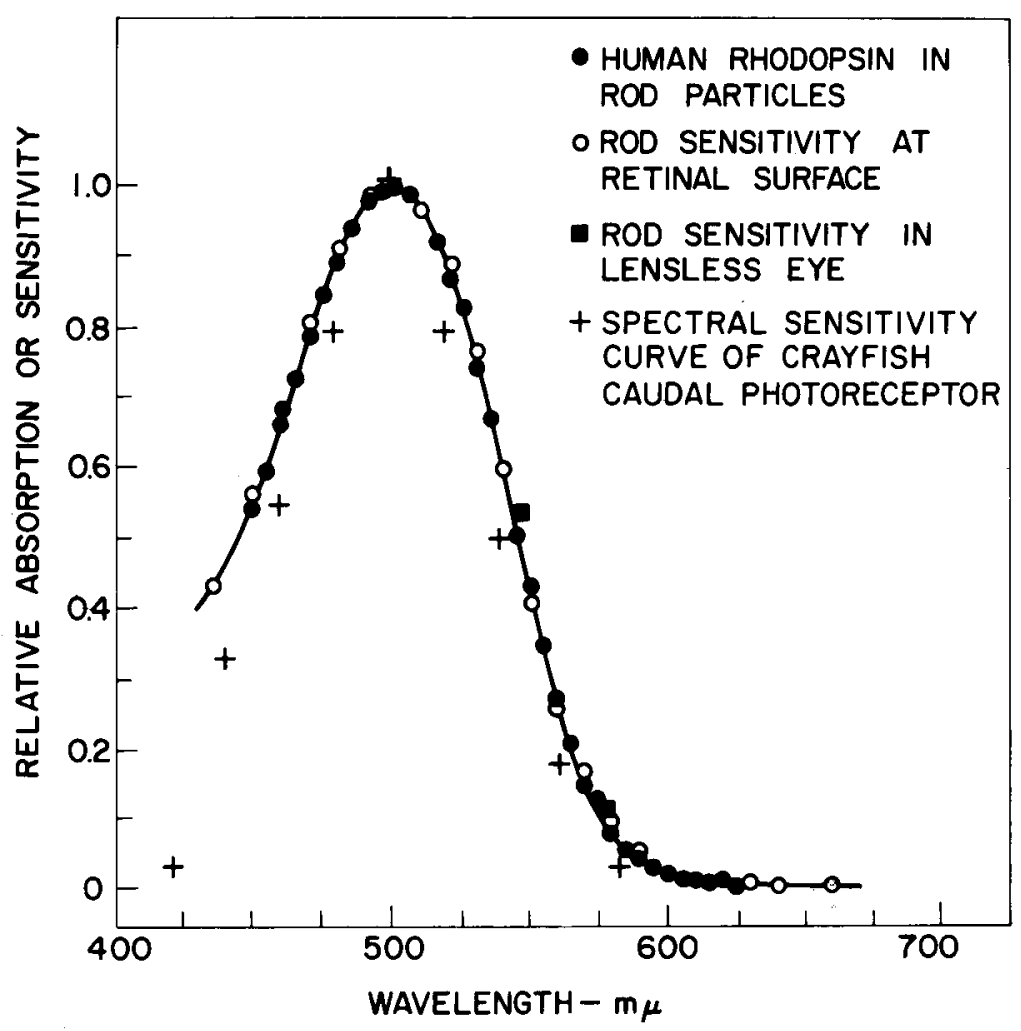

FIGURE 7. The spectral sensitivity curve for the caudal photochemical of the crayfish plotted over the results of three different experiments on humans. (Data for humans are from Wald \& Brown, 1958.)

spectrum and Bruno and Kennedy's (1962) measurement with a threshold criterion.

There appears to be a slightly narrower curve for the results of the present experiment than that found in the Wald data; however, the fact that the peak occurs at $500 \mathrm{~m} \mu$ identifies the crayfish caudal photoreceptor pigment as a rhodopsin (Retinene, plus rod type opsin) similar to that found in human rods.

Another major result of this investigation is to point out the relation between the transformations of the stimulus energy into electrophysiological signals and, what is believed is a relevant phenomenon, the psychophysical function of estimates of apparent magnitudes. Ekman and his colleagues (1960) have done what appears to be the only human psychophysical analog of the current study and that only for photopic vision. Since only the general form is of interest in the current context we can refer to these relations since those of scotopic vision are not yet available. 
It is our contention that the specific results of this experiment suggest that a considerable portion of the psychophysical transfer function relating stimulus intensity and brightness can be attributed to peripheral effects. This suggestion is based upon the fact that the curves for both the human psychophysical data and crayfish electrophysiological data are both strongly negatively accelerated, and very similar in form. FIGURE 8 shows the curves, for their respective peak values, normalized and plotted on the same coordinates. It is not our intention to show mathematical identity (the presence of a general formal psychophysical relation has been challenged by von Mises (1951), but to point out that major portions of the function of stimulus

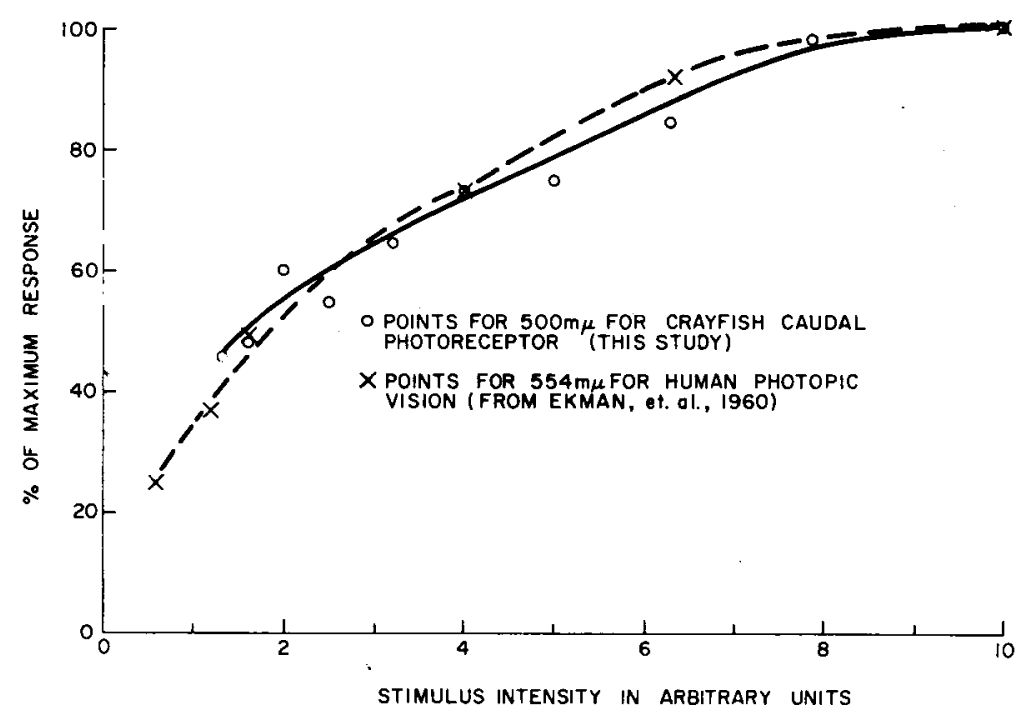

FIGURE 8. A comparison of our results for the rhodopsin-like chemical in the caudal photoreceptor of the crayfish and the human photopic data of Ekman and his colleagues (1960). The crayfish data have been normalized by taking percentages of the maximum value and subtracting spontaneous activity.

compression used to enhance the range of a receptor may well be attributed to the most peripheral element in the afferent chain. In this case it appears to the actual photolysis-generator potential-spike potential generation process in the first order neuron.

\section{B. An Example of Class 2 Data Processing-The Human Somatosensory Evoked Potential}

This work has been described in detail at a previous New York Academy of Sciences meeting and has been published in the Annal of that meeting (Uttal \& Cook, 1964). 


\section{An Example of Class 3 Data Processing-- The Computer Teaching Machine}

Because of the speed, logical power, and memory capacity of modern digital computers it has been possible to simulate a conversational tutorial interaction between a computer and a student. This interaction has been demonstrated to serve as a powerful teaching technique under some conditions by several investigators. One recent paper which is of particular interest to this symposium is by Swets, Feurzeig, Harris, and Marill (1963), and deals with the problem of tutoring diagnostic techniques such as might be taught in a medical school.

The work at our laboratory has dealt with other subject matters, but illustrates fairly well the tutorial techniques which can be marshalled when one is using a general-purpose digital machine.

The general approach has been to develop a computer control program along with appropriate lists of dictionary and conversational items which can be communicated through an appropriate display device to a student. The student's response must also be enterable through an appropriate communication link to the computer for interpretation by the control program. The critical concept is the nonlinear nature of the sequence of events. Each statement and dictionary item, or text book reference is selected on the basis of an analysis of the student's past performance in much the same way as a human conversation adapts to the various responses of the conversants. This closed-loop form of interaction provides each student with a personalized sequence of instruction, keeping motivation high and material relevant, thus effectively carrying out the instructional function.

The three courses which we have selected for our curriculum were chosen as experimental tools rather than working courses. Each represents a much larger body of curriculum courses which have logical similarities to the chosen one. For example, a stenotypy course is the archtype of all of those motor skill courses in which verbal associations are made with motor functions. A German course represents the language course and a statistics course models the mathematical concept type of training.

In some modest pilot studies which we have conducted, reductions in learning time over conventional lecture methods were as great as five to one. Interestingly enough, the computer tutorial methods, because of their adaptive nature, usually can guarantee as high a degree of mastery as may be desired.

Complete details of the programs and pilot studies can be found elsewhere (Uttal, 1962). The following discussion of our stenotypy course* illustrates various features a computer tutorial program may include.

*The collaboration of my colleagues, Mrs. L. Selfridge, Mrs. Anna Maher, and Mrs. Marilyn Charap, in the preparation of this material and the running of the experiment is gratefully acknowledged. 
The computer program to teach stenotypy* is divided into three main sections. The first section assembles an exercise for the student based on the student's record. The second section presents items to the student and grades his responses. The third section analyzes the performance of the student on this past exercise and prepares information to be used in determining his new exercise.

\section{Section 1-Lesson Assembly}

The program starts with the computer interrogating the "I am ready" key. As long as the key is inactive, the computer continues interrogating until a student has indicated he is ready to begin. When the "I am ready" key is depressed, the time is recorded, and the core locations that will contain information on reaction times are cleared. The student record containing detailed information about his past record is read from disc, the disc location having been determined from the student number that was dialed into the auxiliary panel of the stenotypy teaching terminal. If the computer has no student record associated with the given student number, the machine will originate one. Otherwise, the student is welcomed to class by name, and if this is the student's first time on the machine, he is given familiarization practice with the stenotypy teaching terminal before he receives his first formal exercise.

While the computer displays the welcome to the student, the student's new exercise is being determined. The number of items the student receives in one exercise is a function of how much of the special stenotypy training dictionary he has gone through. If he has progressed to the section teaching entire words, he will receive a maximum of 50 items in an exercise; otherwise, he will receive a maximum of 25 items. If the student has not reached item 500 , every new item will be presented with a set of cue lights and he will be told "correct" whenever his answer is right. However, if he is beyond item number 500 , he will not receive cue lights for new items, nor will the reinforcing "correct" be presented. The implicit reinforcement of the presentation of the next item appears to be sufficiently effective for advanced students. Later, however, he will again receive cues on all new items when he starts to learn abbreviations beginning at item 1602 in the master dictionary.

The student's record is periodically examined to determine if he is at a test point. If he is not at a test point at the end of any exercise, a new exercise is assembled, but if he is at a test point, he will be told "Let's have a review" and will be given a review test which must be passed before new material will be presented. If the test is failed, the student will be given additional remedial treatment and retested periodically until he passes the test.

*The stenotypy project was supported in part by the Air Research and Development Command, Rome Air Development Center, Griffis Air Force Base, N. Y., under contract AF 30(602)2466. 
Review tests are given at five specific points in the present program. 1. Test Point 1 follows completion of the keyboard lesson.

2. Test Point 2 follows completion of the lesson containing suffixes and letters of the alphabet that are combinations of two or more keys.

3 . Test Point 3 follows the lesson on numbers.

4. Test Point 4 follows the lesson on voicing of vowels, but the review test contains voicing, numbers, suffixes, and alphabet.

5. Test Point 5 is reached when the student has had some experience with words, and the review test contains voicing of vowels, alphabet, suffixes, and words.

Subsequent review tests are presented every hundred-and-first item, and the review test material is based on all those items which the student has already accomplished.

In a review test, all items are given without cues. If the student makes an error, the item is presented to him with cue lights, immediately correcting the deficiency. If the student answers 90 per cent of the items correctly without any prompts, he is told "you are doing very well," and will be given new material in his next exercise. If the student answers less than 90 per cent of the items correctly, he is told "let's practice some more," and his new exercise will consist of review items heavily weighted with those items that he has had trouble with. No new material will be given. Since reaction times are measured, an alternative test failure criterion can be based upon overly long reaction time as measured by the clock. A test report card is punched immediately following completion of the test which contains the student number, number of correct responses, number of items in the test, percentage of items answered correctly, date, and the average reaction time for correct responses.

An exercise with a maximum of 25 or 50 items is prepared for the student depending on mode (see below) and the number of items the computer has stored in his so-called "error buckets."

Bucket 1 contains all the items of the dictionary that have not been presented to the student, i.e., all new items.

Bucket 2 contains items that the student has missed one time. Bucket 3 contains items that the student has missed two times. Bucket 4 contains items that the student has missed three times. Bucket 5 contains items that the student has missed four times. Bucket 6 contains items that the student has missed five or more times.

Bucket 7 contains the items on which the student has performed successfully.

An item is called accomplished and goes into Bucket 7 when the student has answered it correctly without cue and has not moved the item at any time into the error buckets or when an item is moved by successive correct answers from an error bucket. 
There are two modes of lesson assembly. Exercises are prepared in the error mode when the student has not passed a review test or has made too many previous errors. He will get items chosen successively from all of the items in Buckets $6,5,4,3,2$, and 7 until 25 or 50 items have been chosen.

In the normal mode, the student receives all items that are in Buckets 5 and 6 and one-half of the items in Buckets 2,3, and 4. The remaining number of items in the exercise are distributed between Buckets 7 and 1 , with $5 / 6$ of the remainder coming from Bucket 7 and 1/6 from Bucket 1 . However, the student will not receive items that take him over a test point unless the test has been passed as all new items are scanned to see whether a new lesson block is reached. After passing a test at a test point, the student is told "read your text" and what page to read. Then the machine is automatically placed in the not ready status. When he has completed his text assignment, the student pushes the "I am ready" key and the next exercise is presented.

\section{Section 2-Item Presentation and Grading}

In new lesson assemblies, items taken from Buckets 2 through 6 are always initially presented with cues and abbreviations. Items in Bucket 1 may or may not be presented with cues depending upon their dictionary location and the student's record.

Bookkeeping operations are carried out for each item and a number of informative registers are kept active.

1. When an item is presented to the student, the time is recorded. When the student gives a response, the elapsed time is computed and is added to the total time for responses. A one is added to the "total number of presentations" register.

2. If the student requested a prompt, the elapsed time is added to the total time for prompts and a one is added to the "number of prompts requested" reigster. "Retry" is noted and the item is presented with cues.

3 . If the student stenotyped an answer to the item, the answer is checked. If his answer agrees with the answer in the machine, the elapsed time is added to the total time for correct responses, and a one is added to the "total number of correct responses" register. The student may or may not be told "correct" and "no error" is noted. If the student's answer is incorrect, a check is made to see if there is an acceptable alternate answer. If there is, the student's response is checked against the alternate answer(s). When no more alternate answers exist, the student is told "wrong" and an error card is punched. The error card contains the student number, the items presented, the student's incorrect response, and whether the 
student received the item with cues or not. The elapsed time is added to the total time for incorrect responses, and a one is added to the "total number of incorrect responses" register. "Error" is noted.

The student is thus led through a sequence of items with or without cues which depend specifically upon his past performance.

\section{Section 3-Past Exercise Performance Evaluation}

When the student completes an exercise, he is told "you have completed exercise number xxx." by means of the electroluminescent alphanumeric display. The "exercise number" register is increased by one after each exercise. The only exercise that contains exactly the same material for any two students is exercise number 001 , i.e., the very first exercise. All others will have been individually tailored to meet the needs of the student.

The student is then told to "hit-stop-or-next line" to indicate whether he wishes to stop or to continue.

While these statements are being displayed to the student, the computer is also analyzing the results of the previous exercise. The following changes in the bucket configuration are also being made.

\begin{tabular}{crrr} 
Items from Bucket \# & Marked & Put into Bucket \# \\
\cline { 1 - 2 } 1 & correct & 7 \\
2 & correct & 7 \\
3 & correct & 2 \\
4 & correct & 3 \\
5 & correct & 4 \\
6 & correct & 5 \\
7 & correct & remain in & 7 \\
& incorrect & \\
1 & incorrect & 2 \\
2 & incorrect & 3 \\
3 & incorrect & 4 \\
4 & incorrect & 5 \\
5 & incorrect & remain in \\
6 & incorrect & 6 \\
7 & &
\end{tabular}

Items that were not presented in this exercise remain in the original Bucket 1 . Bucket 7 can contain a maximum of 120 items. When this number is exceeded, the older items are dropped, so that the student moves gradually through the master dictionary.

Buckets 2 through 6 can contain a maximum of 60 items. When one of these error buckets overflows some older items may be dropped but the 
main effect is to put the student into the error mode until he cleans out some of the error buckets.

If the student wishes to continue, a new exercise is prepared for him. However, if the student has depressed the "I would like to stop" key, a session report card is punched containing the following information:

1. Student number

2. Number of responses

3. Average reaction time per response

4. Average reaction time per incorrect response

5. Number of incorrect responses

6. Number of prompts requested

7. Average reaction time per prompt

8. Number of correct responses

9. Average reaction time per correct response

10. Date

11. Number of new items presented

12. The next new item that the student will receive

13. The total time on the machine

The student is then told "that's all for today."

\section{Pilot Study Findings in the Stenotypy Course}

Three secretarial science students from a nearby college were recruited to serve as subjects in our experimental stenotypy course. Although all three were skilled typists, only two of the young ladies had shorthand training.

The problem of comparison of stenotypists trained with a computer method and those trained with conventional methods is a particularly complicated one. There are many reasons which confuse this issue and which suggest that a direct comparison will be difficult, if at all possible, to make. For example, the students trained in this computer course were oriented toward a high level of precision of keying because of the nature of the task for which they were being trained - the real time entry of spoken words into a computer. Our students were constantly sacrificing speed to enhance their accuracy in order to meet the strict standards set by the computer teaching system. It has been observed that conventional stenotypists can make a large number of errors in keying but by making use of the sequential redundancies of the English language are able to retrieve almost all of a given message. Such a flexibility is not yet available in computer transcription systems; and, therefore, our type of training may be exactly what is required for such an automatic transcription system.

The best estimates of conventional training which can be made suggest a 52-week, 700-hour training period to produce students who were able to typically stenotype at a rate of 120 words $/ \mathrm{min}$. Another 52 -week, 800 -hour course brought the students to a 175 -word speed level. Both of these periods 
of time, of course, included transcription time which was not included in our course.

We were able to give each of the students approximately 50 clocked hours of computerized instruction. During that period they completed the entire theory section of the stenotypy course which we estimate would have taken 200-300 hours of conventional instruction.

The computer's data processing capability was used to assemble and statistically analyze a large amount of data during the student training period. We must admit, however, that this abundance of information somewhat overwhelmed us. Although it was an easy task to program the computer to collect these data, it is a massive job to study and interpret all of these measures.

In addition to the abundance of the data, there was another distinction which was extremely important in determining the nature of these results. The computer teaching machine is constantly adapting to the individual student by providing new material as soon as the old is mastered. Thus, there is no static base level, or internal control, against which all other measurements can be standardized. Many of the data which were collected showed the effect of this adaptation by showing only minimal changes after the early exercises. Wide deviations from the usual score were found only when an entirely new class of material, such as abbreviations, was encountered or there was a very long interval between earlier lessons and the current one.

One interesting observation is the difference in reaction times for those items which were keyed correctly and those that were incorrectly entered into the machine. All three subjects show significantly longer reaction times for those items which were missed. This result is obviously not a simple effect of a single variable. Although the subjects did have another response modeasking for a prompt for doubtful items-it is possible that their hesitations on a class of somewhat less doubtful items are being reflected here.

All three students had acquired a vocabulary of approximately 2,000 stenotypy words which could be encoded with an error rate less than 10 per cent for two subjects and less than five per cent for the other.

\section{REFERENCES}

BRUNO, M. S. \& D. KENNEDY. 1962. Spectral sensitivity of photoreceptor neurons in the sixth ganglion of the crayfish. Comp. Biochem. Physiol. 6: 41-46.

COX, D. R. \& W. L. SMITH. 1954. On the superposition of renewal processes. Biometrika 41: 91-99.

DAWSON, G. D. 1950. Cerebral responses to nerve stimulation in man. Brit. Med. Bull. 6: 326-329.

EKMAN, G., H. EISLER \& T. KUNNAPAS. 1960. Brightness scales for monochromatic light. Scand. J. Psychol. 1: 41-48.

HICHAR, J. K. 1959. Pulse height analysis of spontaneous activity in the crayfish nervous system. Medical Electronics. Proc. Second Intern. Conf. Med. Electron.

KENNEDY, D. \& M. S. BRUNO. 1961. The spectral sensitivity of crayfish and lobster vision. J. Gen. Physiol. 44: 1089-1102. 
PROSSER, C. L. 1934. Action potentials in the nervous system of the crayfish. II Responses to illumination of the eye and caudal ganglion. J. Cellular Comp. Physiol. 4: 363-377.

STARK, L. 1959. Transfer function of a biological photoreceptor: Wright Air Develop. Center Rept. : 59-311.

SWETS, J. A., W. FEURZEIG, JUDITH HARRIS \& THOMAS MARILL. 1963. The Socratic System: A Computer System to Aid in Teaching Concepts. Bolt, Berenek, \& Newman, Co. Cambridge, Mass. Rept. No. 1007.

UTTAL, W. R. 1962. My Teacher Has Three Arms. IBM Res. Rept. RC-788.

UTTAL, W. R. 1960. The IBM Biophysical Research System. IBM Res. Rept. RC-195.

UTTAL, W. R. \& L. COOK. 1964. Systematics of the evoked somatosensory cortical potential: A psychophysical-neurophysiological comparison. Ann. N.Y. Acad. Sci. $112(1)$.

UTTAL, W. R., \& H. KASPRZAK. 1962. The caudal photoreceptor of the crayfish: A quantitative study of responses to intensity, temporal and wavelength variables. IBM Res. Rept. RC-620.

UTTAL, W. R. \& P. A. ROLAND. 1961. A terminal device for entry of neuroelectric data into an electronic data processing machine. Electroencephalog. Clin. Neurophysiol. 13: $637-640$.

VON MISES, R. 1951. Positivism. Harvard Univ. Press. Cambridge, Mass.

WALD, G.\& P. K. BROWN. 1958. Human rhodopsin. Science 127: 222-226. 